\title{
Utilização do resíduo da casca de laranja para produção de biossurfactantes por Bacillus subtilis
}

\author{
Use of orange peel waste for production of biosurfactant by Bacillus subtilis
}

\author{
F. Rovina ${ }^{1 *}$; D. D. Ehrhardt ${ }^{2}$; E. B. Tambourgi ${ }^{1}$ \\ ${ }^{1}$ Departamento de Engenharia de Sistemas Químicos/Laboratório de Processos de Separaçao II/Faculdade de \\ Engenharia Química, Universidade Estadual de Campinas, CEP 13083-852, Campinas-SP, Brasil
}

*fernnada.rovina@hotmail.com

(Recebido em 23 de outubro de 2017; aceito em 25 de abril de 2018)

\begin{abstract}
Os biossurfactantes são produzidos naturalmente por microrganismos e podem ser sintetizados a partir de substratos renováveis. Possuem alta biodegradabilidade e baixa toxidade, porém a produção de biossurfactante tem sido limitada devido aos altos custos de produção e purificação. O presente trabalho teve como objetivo a produção de biossurfactante por Bacillus subtilis em meio de fermentação a base de resíduos do processamento da casca da laranja nas diluições de $60 \%$ e $40 \%$, a $37^{\circ} \mathrm{C}$ durante 24 horas. Foram realizados testes de tensão superficial e índice de emulsão para comprovação da ação tensoativa do biossurfactante produzido. A partir do método do peso da gota, a fermentação realizada com substrato diluído em $40 \%$, obteve uma redução da tensão superficial de $25 \%$, após 24 horas de fermentação e foi observado o maior índice de emulsificação após 7 horas de fermentação, chegando a 29 \% quando utilizado o querosene e $11 \%$ com o óleo de soja. Portanto, os resultados mostraram que os resíduos do processamento da casca da laranja é um potencial substrato na produção de biossurfactantes utilizando o Bacillus subtilis.

Palavras-chave: Biossurfactante, produção de biossurfactantes, Bacillus subtilis.
\end{abstract}

The biosurfactants are naturally produced by microorganisms and can be synthesized from renewable substrates. They have high biodegradability and low toxicity, however the biosurfactants production has been limited due to high costs of production and purification. This study aimed to produce biosurfactant by Bacillus subtilis at $37^{\circ} \mathrm{C}$ for 24 hours, using the residue from processing of in orange peel in the dilutions of $60 \%$ and $40 \%$. Tests were performed of surface tension and index of emulsion for attesting the action tensioactive of the biosurfactant produced. From the drop weight method, the fermentation held $40 \%$ diluted in substrate, obtained a reduction of surface tension of $25 \%$ after 24 hours of fermentation. This fermentation obtained the highest percentage of emulsification after 7 hours of fermentation, reaching $29 \%$ when used kerosene and $11 \%$ with soybean oil. Therefore, the results showed that the waste from the processing of orange peel is a potential substrate for the production of biosurfactants using Bacillus subtilis.

Keywords:Biosurfactant, biosurfactants production, Bacillus subtilis.

\section{INTRODUÇÃO}

Surfactantes e biossurfactantes são moléculas anfipáticas que possuem propriedades hidrofílicas e hidrofóbicas. A porção hidrofílica pode ser iônica, não-iônica ou anfotérica, enquanto que a extremidade hidrofóbica é frequentemente constituída por uma cadeia hidrocarbonada [1]. Em função da estrutura molecular, os surfactantes tendem a alterar as propriedades interfaciais e superficiais dos fluidos ocasionando a capacidade de detergência, emulsificação, lubrificação, capacidade espumante e solubilização [2].

Dentre as aplicações dos biossurfactantes se destacam a redução da tensão superficial, o poder emulsificante, transporte de hidrocarbonetos e aderência/liberação da célula de superfícies pela excreção de biossurfactantes, atividade antibiótica e biorremediação de solos contaminados [3]. Além disso, os biossurfactantes possuem baixa toxidade, podendo ser aplicado nas indústrias de alimentos, cosméticos e fármacos [4,5]; sendo classificados em glicolipídeos, lipossacarídeos, lipopeptídeos/lipoproteínas, fosfolipídeos e ácidos graxos/lipídeos neutros, além de surfactantes poliméricos e surfactantes particulados [6]. Entre os lipopeptídeos e lipoproteínas, estão a surfactina, polimixina, octapeptinas, fengicinas e iturinas, as quais possuem efeitos antibióticos e alta capacidade na redução da tensão superficial $[7,8]$. 
A surfactina produzida pelo Bacillus subtilis, está entre os mais efetivos biossurfactantes devido a sua atividade antibiótica e excepcional atividade superficial, reduzindo a tensão superficial da água $\left(20^{\circ} \mathrm{C}\right)$ de 72 para $35 \mathrm{mN} / \mathrm{m}[2,9]$. Os métodos mais empregados para detectar a produção de biossurfactantes são o índice de emulsão e a medição da tensão superficial [10]. O índice de emulsão é analisado pela altura da emulsão em comparação com a altura total da mistura de biossurfactante e óleo, após $24 \mathrm{~h}$ de repouso [2,11]. Enquanto que, a tensão superficial surge nos líquidos como resultado do desequilíbrio entre as forças que agem sobre as moléculas da superfície em relação àquelas que se encontram no interior da solução, a mensuração da tensão superficial pode ser realizada utilizando os tensiômetros, que calculam a razão entre a força aplicada pelo líquido observado e a área utilizada para transportar a molécula à superfície [10,12]. Outro método satisfatório e simples empregado para medir a tensão superficial é o peso da gota [12].

Quando comparados com os surfactantes sintéticos, os compostos de origem microbiana possuem as vantagens de possuírem baixa toxicidade, melhor degradabilidade, e tolerância à altas temperaturas e de $\mathrm{pH}$ [13]. No entanto, a eficiência na produção do biossurfactante está relacionada ao desenvolvimento de um processo que utilize materiais de baixo custo, forneça alto rendimento e seja apropriado para ser utilizado industrialmente $[2,10]$. Para a ampliação das possibilidades de aplicação do biossurfactante, o uso de resíduos industriais ou orgânicos como meio de cultivo é uma alternativa viável, uma vez que a matéria-prima representa cerca de $30 \%$ de custos totais em um processo biotecnológico [2,14].

Barros et al. (2008) [2] produziram biossurfactante utilizando a linhagem LB5a de Bacillus subtilis, cultivado em manipueira. Os resultados mostraram que o biossurfactante exibiu uma excelente atividade tensoativa, reduzindo a tensão superficial para $27,01 \mathrm{mN} / \mathrm{m}$ e obtiveram elevados índices de emulsão durante o período de 24 horas, em torno de 76,4 \% utilizando o diesel e demonstraram a importância do biossurfactante possuir um alto grau de pureza para potenciais aplicações em diversos ramos industriais, tais como cosméticos e alimentos.

As variações entre os rendimentos da produção de surfactina se deve principalmente às condições de processo e/ou nutricionais $[3,6]$. Os fatores de crescimento para os microrganismos geralmente não são conhecidos e por isso microbiologistas adicionam compostos complexos como extrato de levedura e peptona para garantir tais suprimentos [15].

Diversos estudos estão voltados para a otimização das condições de cultivo, como a proporção e tempo de incubação do inóculo, temperatura, agitação ou a combinação desses fatores $[2,16,17]$. Os substratos utilizados como fonte de nutrientes que alteram a produção de biossurfactantes incluem carbono, nitrogênio, fosfatos, íons metálicos e outros componentes [3]. Os melhores substratos para o crescimento microbiano são os resíduos ricos em carboidratos ou lipídeos, com adequada relação carbono/nitrogênio e que apresentem altas concentrações de micronutrientes importantes para o metabolismo microbiano [9].

Alguns resíduos já foram testados como substrato, dentre eles estão as cascas de frutas, como abacaxi, caju e maçã, o melaço, manipueira, soro de leite e rejeitos do processamento de óleos $[1,3,7,14,18,19]$. A produção de laranja ultrapassa 80 milhões de toneladas/ano, em média $34 \%$ da produção é transformada em suco, mas em grandes países produtores como o Brasil e Estados Unidos, esta percentagem chega a 90\%, o que gera grande quantidade de resíduos [20]. Os resíduos sólidos da laranja que são representados pela casca, polpa e os resíduos líquidos, são ricos em óleos essenciais, pectina, açúcares, ácidos orgânicos e sais [21]. Alguns estudos destacam os resíduos da laranja, em especial a sua casca, como um promissor substrato para o desenvolvimento de microrganismos [22, 23].

Ahmed et al. (2016) [22] utilizaram resíduos da casca da laranja como fonte de carbono no processo fermentativo para a produção da enzima com Aspergillus niger. Após a análise físicoquímica detectaram alguns compostos presentes na casca da laranja, $7 \%$ de pectina, 6,4\% de lignina, $14,08 \%$ de açúcares totais e 3,7 \% de açucares não redutores e obtiveram resultados satisfatórios para a produção de enzimas.

Tessaro et al. (2010) [23] estudaram a fabricação de vinagre a partir da fermentação da Saccharomyces cerevisiae utilizando como substrato a laranja pera com diferentes correções de açucares do suco obtido. Os resultados mostraram que a laranja, pode ser empregada nos processos fermentativos, sendo que o suco com correção do Brix de $22 \%$ apresentou as melhores condições fermentativas. 
Sendo o Brasil um dos maiores produtores mundiais de laranja, torna a produção de biossurfactante viável tanto pela redução do custo de produção como pela redução dos resíduos. Portanto, o objetivo deste trabalho consiste na produção de biossurfactante por Bacillus subtilis, utilizando o resíduo do processamento da casca da laranja pera, Citrus sinensis (L.) Osbeck, como meio de cultivo.

\section{MATERIAL E MÉTODOS}

\subsection{Microrganismo}

A cepa de Bacillus subtilis utilizada neste trabalho é proveniente do núcleo de cultura do CPQBA- Centro Pluridisciplinar de Pesquisas Químicas, Biológicas e Agrícolas.

Foram preparados 10 tubos de repique do Bacillus subtilis com ágar nutriente, autoclavados por 15 minutos e deixados em repouso até esfriar. O repique foi realizado com auxílio da alça de platina e bico de Bunsen. Os tubos repicados ficaram incubados por 24 horas e mantidos refrigerados $[15,17]$.

As cepas do Bacillus subtilis foram preservadas a $4^{\circ} \mathrm{C}$ com o objetivo de diminuir o metabolismo celular e garantir o controle microbiano [15,17]. Para que houvesse a manutenção e a conservação dos microrganismos, a cultura do Bacillus subtilis foi repicada a cada 30 dias.

\subsection{Produção de Biossurfactante}

O Bacillus subtilis, normalmente, produz a surfactina em temperaturas de $25{ }^{\circ} \mathrm{C}$ a $37^{\circ} \mathrm{C}$ [2,3,15,17]. De acordo com Sen e Swaminathan [17], a temperatura ideal para a produção de surfactina por Bacillus subtilis é $37,4{ }^{\circ} \mathrm{C}$. Neste trabalho, o processo de fermentação para produção de biossurfactante foi realizado com auxílio de um shaker, de agitação pré-fixada, a temperatura constante de $37^{\circ} \mathrm{C}$.

\subsubsection{Preparo do Inóculo}

O preparo do pré-inóculo foi baseado na metodologia de Ehrhardt, 2015 [18].

Inicialmente, foi preparado o pré-inóculo contendo uma solução aquosa de $15 \mathrm{~mL}$ com peptona, cloreto de sódio, extrato de levedura e glicose, foi preparada em um Erlenmeyer de $125 \mathrm{~mL}$, em seguida, autoclavada e resfriada para o meio de cultura ser inoculado. O Erlenmeyer foi colocado em um shaker, com agitação constante e temperatura de $37^{\circ} \mathrm{C}$, durante 6 horas.

Após o período de 6 horas descrito, uma porção de $10 \mathrm{~mL}$ do pré-inóculo, já adaptado ao meio de crescimento, foi transferida para uma solução de $100 \mathrm{~mL}$ contendo peptona, cloreto de sódio, extrato de levedura e glicose e mantida em shaker, com agitação constante de $150 \mathrm{rpm}$ e temperatura de $37^{\circ} \mathrm{C}$, durante 24 horas, para garantir o desenvolvimento do microrganismo no meio.

A padronização do inóculo foi realizada utilizando $100 \mathrm{~mL}$ do mesmo meio nutriente ajustado em espectrofotômetro em uma faixa de absorbância de 0,08 a 0,1 , com comprimento de onda de $660 \mathrm{~nm}$. Foram mantidas em todas as fermentações as mesmas concentrações contendo $(\mathrm{p} / \mathrm{v}) \mathrm{de}$ peptona, $0,76 \%$, cloreto de sódio, $0,06 \%$, levedura $1 \%$ e glicose, $5 \%$, (p/v), que são consideradas por diversos estudos como ótimas para o desenvolvimento do Bacillus subtilis.

\subsubsection{Preparo do Meio de Cultura}

Em seis frascos de $250 \mathrm{~mL}$ foram adicionados cerca de $80 \mathrm{~mL}$ de caldo proveniente da centrifugação das cascas de laranja, variando as diluições em $60 \%$ e $40 \%$ de água após alguns testes. Os frascos contendo o substrato foram previamente autoclavados a $121^{\circ} \mathrm{C}$, por 10 minutos. As condições de esterilização foram definidas de acordo com a metodologia de Ehrhardt, 2015 [18]. 


\subsubsection{Fermentação}

As fermentações foram realizadas em seis frascos de $250 \mathrm{~mL}$, contendo o substrato anteriormente descrito. Em cada frasco foram adicionados $8 \mathrm{~mL}$ do inóculo $(10 \%)$, devidamente fechados com tampas de gaze e algodão, para evitar contaminação e mantidos sob agitação constante, à $37^{\circ} \mathrm{C}$ por 24 horas [17].

Durante este período, foram retiradas 7 amostras, uma inicial, uma segunda após 1 hora de fermentação, as 4 seguintes em intervalos de 2 horas e a última amostra após 24 horas de fermentação. A amostra do ponto inicial de fermentação foi utilizada como controle, ou seja, um ponto de comparação em relação às amostras retiradas durante o processo fermentativo.

As amostras foram centrifugadas a $3.000 \mathrm{rpm}$ por 10 minutos e submetidas à determinação de tensão superficial e índice de emulsificação [18]. As fermentações foram realizadas em duplicata, para cada diluição do substrato testado, ou seja, $60 \%$ e $40 \%$ em água.

\subsection{Medida da Tensão Superficial}

A tensão superficial foi medida utilizando a metodologia do Peso da Gota baseado em Behring (2004) [12].

O método do Peso da Gota consiste no preparo de uma bureta fixada a um suporte universal e acoplada a um Erlenmeyer, de forma que a parte inferior à torneira fique toda dentro do recipiente coletor, evitando a influência de correntes de ar sobre a formação da gota. A queda da gota foi ajustada em intervalos de 1 minuto para determinar a massa e o raio da circunferência da gota e posteriormente calcular a tensão superficial, com isso foi possível comparar a tensão das amostras com a da água igual a $72 \mathrm{mN} / \mathrm{m}$ a $25^{\circ} \mathrm{C}$. Os experimentos foram realizados em duplicata.

\section{4. Ïndice de Emulsão (E24)}

O índice de emulsão foi medido através da proporção óleo/biossurfactante, de acordo com a metodologia proposta por Cooper e Goldenberg (1987) [16].

Foram testados o óleo de soja e querose. Em cada tubo de ensaio foram adicionados $1 \mathrm{~mL}$ de óleo e, em seguida, $1 \mathrm{~mL}$ do biossurfactante produzido em tubos graduados. Os tubos foram agitados em vortéx por um minuto e deixados em repouso por 24 horas. Após 24 horas foi realizada a análise do índice de emulsão através da relação entre a altura da camada emulsificada com a camada total, conforme a seguinte equação:

$$
\%(E 24)=\frac{\text { altura da Emulsão }}{\text { altura total }}
$$

\section{RESULTADOS E DISCUSSÃO}

\subsection{Tensão Superficial}

A análise da tensão superficial é um dos indicativos da produção de biossurfactantes. A partir da técnica do peso da gota, foi obtida a redução da tensão superficial ao longo do tempo das fermentações e da quantidade de nutrientes fornecido ao microrganismo. A análise realizada utilizando 5\% de glicose no preparo do pré-inóculo, alcançou resultados expressivos quanto a redução da tensão superficial ao longo do tempo das fermentações.

Na primeira fermentação, a amostra no tempo inicial apresentou uma tensão superficial de 58,11 $\mathrm{mN} / \mathrm{m}$, o qual foi diminuindo ao longo do tempo até que se alcançou o valor de $48,65 \mathrm{mN} / \mathrm{m}$ após 24 horas de fermentação, o que gerou uma redução de $16,29 \%$ da tensão superficial, conforme mostrado na Figura 1. 


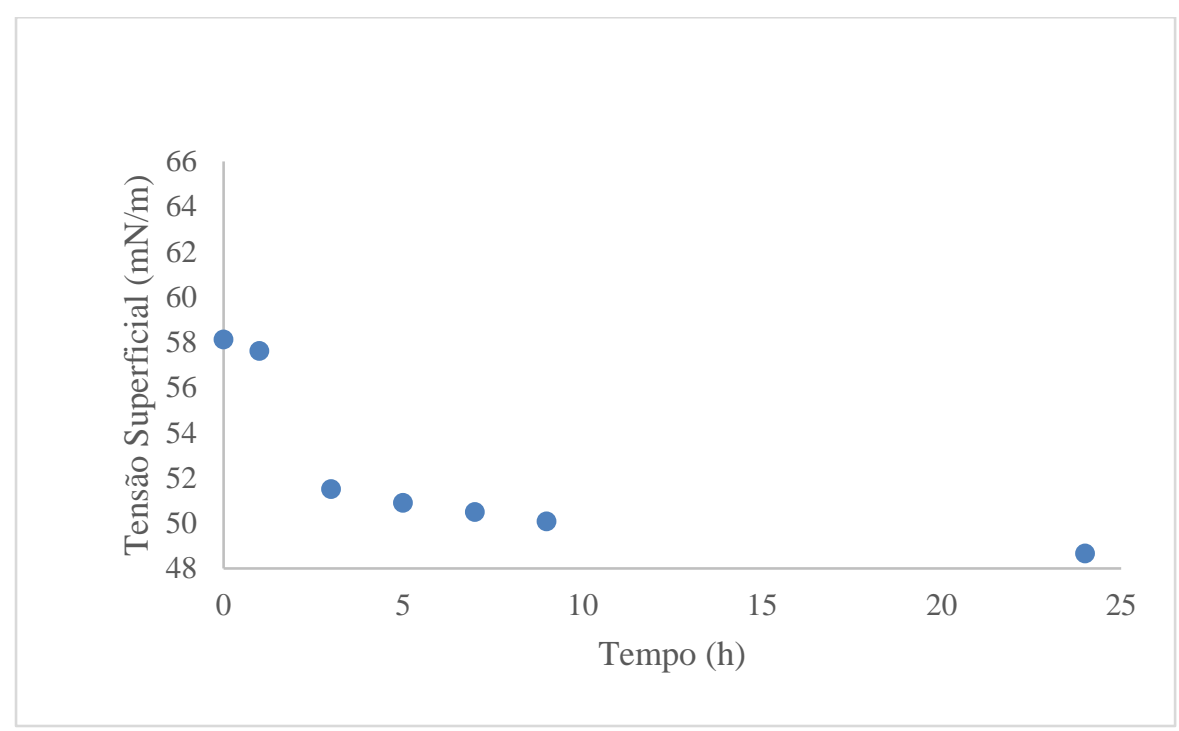

Figura 1: Análise da tensão superficial ao longo do tempo, com substrato diluído em $60 \%$.

A baixa redução da tensão superficial ao longo do tempo pode ser explicada pelo substrato utilizado nesta fermentação estar diluído em $60 \%$ de água o que implicou em uma baixa tensão superficial no meio de cultivo estéril (tempo zero) e, portanto, os resultados ao longo das 24 horas de fermentação passaram a não ser significativos. Em geral, o aumento da concentração dos substratos com altos níveis de carboidratos, nitrogênio ou lipídeos suprem a necessidade de fonte de carbono dos biossurfactantes $[9,19]$.

Rane et al. (2017) [24] investigaram o potencial do Bacillus subtilis, crescer em diferentes fontes de carbono. $\mathrm{O}$ biossurfactante produzido em meio contendo glicose como única fonte de carbono obteve a concentração de açúcar total de $0,207 \mathrm{~g} / \mathrm{l}$ e o mesmo com melaço como fonte de carbono foi de $0,241 \mathrm{~g} / \mathrm{l}$. Entre os extratos de resíduos agrícolas, a casca de laranja obteve a quantidade de açúcar total de $0,089 \mathrm{~g} / \mathrm{l}$, resultado superior ao encontrado nas cascas de banana, que obteve 0,049 $\mathrm{g} / \mathrm{l}$.

Foram realizadas novas fermentações com o substrato diluído em $40 \%$ de água, obtendo uma maior redução da tensão superficial, $25,22 \%$, de $65,83 \mathrm{mN} / \mathrm{m}$ para 49,23 mN/m após 24 horas de fermentação. Os resultados obtidos após 24 horas de fermentação são mostrados na Figura 2.

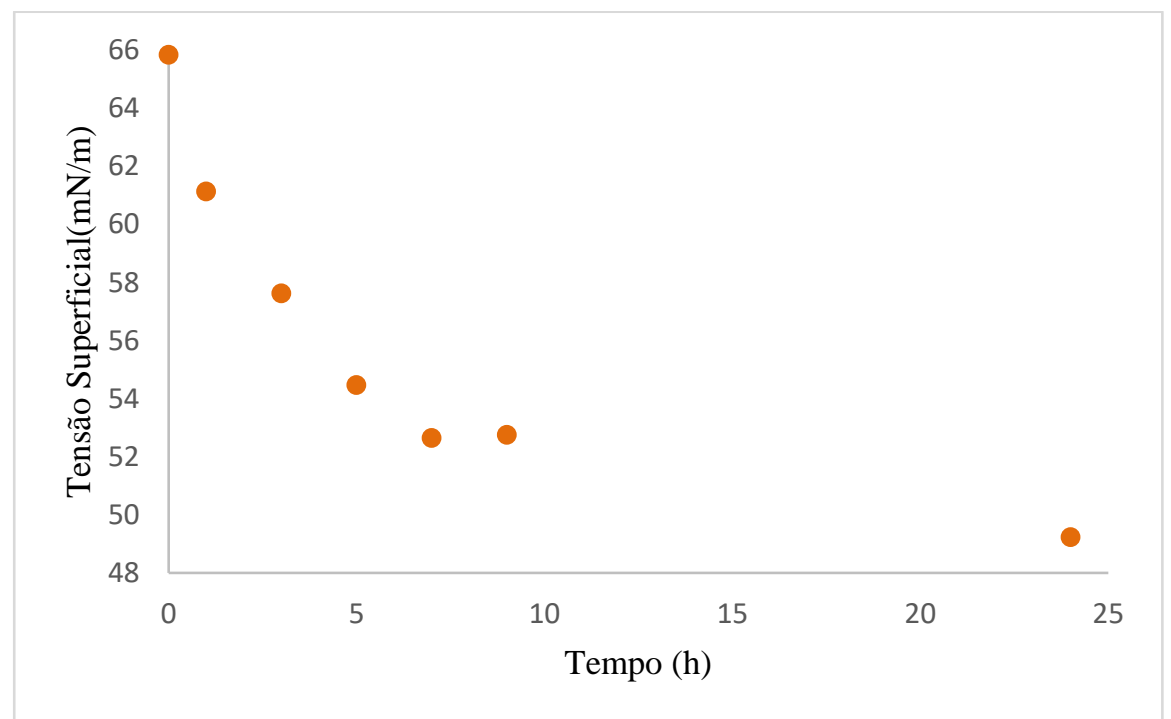

Figura 2: Análise da tensão superficial ao longo do tempo, com substrato diluído em 40\%.

A alta redução da tensão superficial nas primeiras 7 horas de fermentação e a estabilização da redução desse parâmetro ao longo do tempo pode ser devido ao consumo dos nutrientes imediato 
pelo microrganismo, resultando no desenvolvimento do microrganismo nas primeiras horas de fermentação. Alguns estudos indicam que a tensão superficial tem que ser reduzida em valores maiores de $20 \%$ para que o biossurfactante seja considerado ativo [24]. Testes utilizando a suplementação com glicerol e sacarose demonstraram a capacidade de diminuição da tensão superficial alcançando valores de $27 \mathrm{mN} / \mathrm{m}$ [7].

Bezerra (2012) [19], produziu biossurfactante a partir de Pseudomonas aeruginosa utilizando como substrato a manipueira. Para efeito comparativo, o meio diluído em (1:1) obteve $25 \%$ de redução da tensão superficial e o meio suplementado com nutrientes reduziu $30 \%$ após 24 horas de fermentação.

Rane et al. (2017) [24] otimizaram a produção de biossurfactantes por Bacillus subtilis utilizando cascas de laranja e obtiveram máximo rendimento do tensoativo de $0,513 \mathrm{~g} / \mathrm{l} \mathrm{em}$ meio contendo melaço a $4 \%$ e a mais adequada fonte de nitrogênio foi o citrato de amônio férrico. Enquanto, Bueno (2008) [25] testou diferentes fontes de carbono (glicose, sacarose, manitol, frutose e caldo de cana) nas concentrações 1, 2, 3, 4 e $5 \%$ para a produção de biossurfactante por Bacillus subtilis. Os resultados obtidos mostraram que o meio de cultivo rico em sacarose e glicose obtiveram maiores reduções superficiais.

Apesar de um aumento na redução da tensão superficial, a adição de sais comerciais, fontes de nitrogênio e carbono ao substrato poderia afetar o custo do processo e também anular a utilização de resíduos orgânicos como fonte de nutrientes.

\section{2. Índice de Emulsão}

Devido a característica anfifílica, os biossurfactantes apresentam a capacidade de formar emulsões e estabilizá-las. O óleo de soja e o querosene foram avaliados neste estudo. A tabela 1 apresenta o índice de emulsão do biossurfactante produzido utilizando o substrato diluído em 40 $\%$.

Tabela 1: Índice de emulsão de acordo com a proporção óleo/ biossurfactante

\begin{tabular}{cccc}
\hline & & \multicolumn{2}{c}{ Índice de Emulsão } \\
\cline { 3 - 4 } Amostra & Tempo (h) & Óleo de Soja (\%) & Querosene (\%) \\
\hline $\mathbf{1}$ & 1 & - & 10,53 \\
$\mathbf{2}$ & 3 & 5,00 & 10,53 \\
$\mathbf{3}$ & 5 & 5,26 & 16,67 \\
$\mathbf{4}$ & 7 & 10,53 & 29,41 \\
$\mathbf{5}$ & 9 & 10,53 & 23,53 \\
$\mathbf{6}$ & 24 & 5,56 & 10,53 \\
\hline
\end{tabular}

A partir do teste de índice de emulsão, verificou que houve a formação de emulsão, no entanto, foram obtidos valores inferiores a $30 \%$, utilizando tanto óleo de soja como o querosene. O baixo índice de emulsão pode estar relacionado com o tempo de agitação, a quantidade ou a suplementação de nutrientes no preparo da cultura, os óleos testados e a função fisiológica dos emulsionantes microbianos.

Soares et al. (2014) [1] estudaram a produção de biossurfactante a partir do Bacillus subtilis utilizando suco de caju clarificado como fonte de nutriente. Os resultados mostraram que o biossurfactante produzido apresentou índice de emulsão superior a $50 \%$ e concentração micelar crítica de 17,3 mg/L. Enquanto, Pereira et al. (2013) [26] utilizaram diferentes fontes de carbono e nitrogênio como meio de cultivo para a produção de biossurfactantes a partir de três variações do Bacillus subtilis. Entre as fontes de carbono avaliadas, acetato de sódio, citrato de sódio, frutose, glicose, glicerol, n-hexadecano, lactose, extrato de carne, parafina, sacarose, triptona e extrato de leveduras, a sacarose e a glicose obtiveram os maiores índices de emulsão para os três isolados, cerca de $39 \%$.

Sobrinho et al. (2008) [27] obtiveram os índices de emulsão, utilizando biossurfactante produzido a partir de Streptomyces sp, de 47, 41, 36 e $30 \%$ para óleo de milho, óleo de soja, óleo de canola e óleo de girassol, respectivamente. De acordo com Sobrinho et al. (2008) [27], os 
microrganismos produtores de tensoativos podem ser divididos em dois grupos: o primeiro que produz biossurfactante com baixo peso molecular, mas usualmente não são capazes de formar emulsões estáveis e o segundo grupo que produz polímeros que primariamente atuam como estabilizantes de emulsões, mas não afetam a tensão superficial.

Portanto, o biossurfactante produzido neste trabalho foi capaz de reduzir a tensão superficial a níveis aceitáveis, mas quanto a capacidade emulsificante obteve valores abaixo do esperado, o que pode ser devido a não utilização de suplementação nutricional do substrato ou a características morfológicas do microrganismo utilizado.

\section{CONCLUSÃo}

A utilização de substratos renováveis para a produção de biossurfactantes é uma alternativa viável, além de uma destinação aos resíduos orgânicos, contribui para a diminuição do custo de produção, visto que o uso de matéria prima de baixo custo representa cerca de $30 \%$ do custo total do processo.

A partir dos dados obtidos experimentalmente, foi observado que o houve a produção de biossurfactantes por Bacillus subtilis, utilizando os resíduos da casca da laranja, e que este se mostrou efetivo na redução da tensão superficial.

Os testes de índice de emulsão não se apresentaram favoráveis tanto utilizando óleo de soja como querosene, porém estudos apontam que o baixo índice de emulsificação pode estar ligado a linhagem do microrganismo, função fisiológica e que possíveis suplementações ao substrato poderiam aumentar o poder emulsificante deste tensoativo.

Portanto, este trabalho evidenciou a capacidade da produção de biossurfactantes por Bacillus subtilis utilizando o resíduo do processamento da casca da laranja, mas torna- se necessário aprimorar a produção do biossurfactante para obter maiores reduções na tensão superficial e realizar novos testes de emulsão.

\section{REFERÊNCIAS BIBLIOGRÁFICAS}

1. Soares DWF. Produção e Caracterização de Biossurfactantes Obtidos por Linhagens de Bacillus sp. Isolados de Estações de Tratamento de Águas Residuais e de Solo de Manguezais (Ceará- Brasil) [tese de doutorado]. Ceará (CE): Universidade Federal do Ceará; 2014. 162 p.

2. Barros FFC, Quadros CP, Pastore GM. Propriedades Emulsificantes e Estabilidade do Biossurfactante Produzido por Bacillus subtilis em Manipueira. Ciência e Tecnologia de Alimentos. 2008 OutDez;28(4):979-985, doi:10.1590/s0101-20612008000400034.

3. Nitschke M, Pastore GM. Biossurfactantes: Propriedades e Aplicações. Química Nova. 2002 SetOut;25(5):772-776, doi:10.1590/S0100-40422002000500013.

4. Ferreira A, Vecino X, Ferreira D, Cruz JM, Moldes AB, Rodrigues LR. Novel cosmetic formulations containing a biosurfactant from Lactobacillus paracasei. Colloids Surf B Biointerfaces. 2017 Jul;155:522-529, doi:10.1016/j.colsurfb.2017.04.026.

5. Basit M, Rasool MH, Naqvi SAR, Waseem M, Aslam B. Biosurfactants production potential of native strains of Bacillus cereus and their antimicrobial, cytotoxic and antioxidant activities. Pak J Pharm Sci. 2018 Jan;31(1(Suppl.)):251-256.

6. Barros FFC, Quadros CP, Maróstica Junior MR, Pastore GM. Surfactina: Propriedades Químicas, Tecnológicas e Funcionais para Aplicações em Alimentos. Química Nova. 2007 Jan;30(2):409-414, doi:10.1590/s0100-40422007000200031.

7. Zhang J, Xue Q, Gao H, Lai H and Wang P (2016). Production of lipopeptide biosurfactants by Bacillus atrophaeus 5-2a and their potential use in microbial enhanced oil recovery. Microb. Cell Fact. 2016;15(1):168-179, doi:10.1186/s12934-016-0574-8.

8. Daas MS, Jeella ZA, Albert RRR, Fabini DO, Béla R, Jing ZFN, Rebecca JC, Salima KG, John C V. Bacillus amyloliquefaciens ssp. plantarum F11 isolated from Algerian salty lake as a source of biosurfactants and bioactive lipopeptides. FEMS Microbiology Letters. 2018 Jan;1:365(1), doi:10.1093/femsle/fnx248.

9. Nitschke M, Pastore GM. Production and Properties of a Surfactant Obtained from Bacillus subtilis Grown on Cassava Wastewater. Bioresource Technology. 2006 Jan;97(2):336-341, doi:10.1016/j.biortech.2005.02.044. 
10. Rufino RD, Luna JM, Campos-Takaki GM, Sarubbo LA. Characterization and properties of the biosurfactant produced by Candida lipolytica UCP 0988. Electronic Journal of Biotechnology. 2014;17:34-38, doi:10.1016/j.ejbt.2013.12.006

11. Youssef NH, Duncana KE, Naglea DP, Savagea KN, Knappb RM, McInerneya. Comparison of methods to detect biosurfactant production by diverse microorganisms. Journal of Microbiological Methods. 2004 Mar;56(3):339-347, doi:10.1016/j.mimet.2003.11.001.

12. Behring JL. Adaptação no Método do Peso da Gota para Determinação da Tensão Superficial: um Método Simplificado para a Quantificação da CMC de Surfactantes no Ensino da Química. Química Nova. 2004 Mai-Jun;27(3):492-495, doi:10.1590/s0100-40422004000300021.

13. Murugan T, Murugan M, Wins JJA. Screening, Production and Antibacterial Potential of Biosurfactant from Bacillus subtilis Isolated from oil Contaminated Soil. Journal of Applied Pharmaceutical Science. 2016 May-Jun;38(2):115-118.

14. Oliveira DWF, Sousa JR, França WL, Felix KN, Martins, JJL, Gonçalves LRB. Kinetic Study of Biosurfactant Production by Bacillus subtilis LAMI005 grown in Clarified Cashew Apple Juice. Journal of Biotechnology. 2010 Nov;150:421-422, doi:10.1016/j.jbiotec.2010.09.580.

15. Andrade CJ. Estudo da Utilização do Glicerol Oriundo da Cadeia de Biodiesel por Bacillus subtilis para a Produção de Biossurfactantes, Enzimas e Aromas [tese de mestrado]. Campinas (SP): Universidade Estadual de Campinas. Faculdade de Engenharia de Alimentos; 2011. 140 p.

16. Cooper DG, Goldenberg BG. Surface-Active Agents from Two Bacillus Species. Appl Env Microbiol. 1987 Feb;53(2):224-229, doi:0099-2240/87/020224-06\$02.00/0.

17. Sen R, Swaminathan T. Characterization of concentration and purification parameters and operating conditions for the small-scale recovery of surfactin, Process Biochem. 2005 Jan;40:2953-2958, doi: 10.1016/j.procbio.2005.01.014.

18. Ehrhardt DD. Produção de Biossurfactantes por Bacillus subtilis Utilizando Resíduo do Processamento do Abacaxi como Substrato. [tese de mestrado]. Campinas (SP): Universidade Estadual de Campinas. Faculdade de Engenharia Química; 2015. 72 p.

19. Bezerra MS. Estudo da Produção de Biossurfactantes sintetizados por Pseudomonas aeruginosa AP029GVIIA Utilizando Manipueira como Fonte de Carbono. [tese de doutorado]. Natal (RN): Universidade Federal do Rio Grande do Norte. Departamento de Engenharia Química; 2012. 114 p.

20. Alexandrino AM, Fariall HG, Souza CGM, Peralta RM. Aproveitamento do resíduo de laranja para a produção de enzimas lignocelulolíticas por Pleurotusostreatus (Jack:Fr). Ciência e Tecnologia de Alimentos. 2007 Abr-Jun;27(2):364-368.

21. Santos CM, Dweck J, Viotto RS, Rosa AH, Morais LC. Application of orange peel waste in the production of solid biofuels and biosorbents. Bioresource Technology. 2015 Aug;196:469-479, doi:10.1016/j.biortech.2015.07.114.

22. Ahmed I, Zia MA, Hussain MA, Zain A, Naveed MT, Nowrouzi A. Bioprocessing of citrus waste peel for induced pectinase production by Aspergillus niger; its purification and characterization. Journal of Radiation Research and Applied Sciences. 2016 Apr;9(2):148-154, doi:10.1016/j.jrras.2015.11.003.

23. Tessaro D, Larsen AC, Dallago RC, Damasceno SG, Sene L, Coelho SRM. Avaliação das fermentações alcoólica e acética para produção de vinagre a partir de suco de laranja. Acta Scientiarum Technology. 2010;32(2):201-205, doi:10.4025/actascitechnol.v32i2.4275.

24. Rane AN, Baikar VV, Ravi KV, Deopurkar RL. Agro-Industrial Wastes for Production of Biosurfactant by Bacillus subtilis ANR 88 and Its Application in Synthesis of Silver and Gold Nanoparticles. Frontiers in Microbiology. 2017 Mar;8:492, doi:10.3389/fmicb.2017.00492.

25. Bueno SM. Bactérias Produtoras de Biossurfactantes: Isolamento, Produção, Caracterização e Comportamento num Sistema modelo. [tese de doutorado]. São José do Rio Preto (SP): Universidade Estadual Paulista. Istituto de Biociências, Letras e Ciência Exatas; 2008. 89 p.

26. Pereira JFB, Gudiña EJ, Costa R, Votorino R, Teixeira JA, Coutinho JAP, Rodrigues LR. Optimization and Characterization of biosurfactant production by Bacillus subtilis isolates towards microbial enhanced oil recovery applications. Fuel. 2013 May;111:259-268, doi:10.1016/j.fuel.2013.04.040.

27. Sobrinho HBS, Rufino RD, Luna JM, Salgueiro AA, Campostakaki GM, Leite LFC, Sarubbo LA. Utilization of two agroindustrial by products for the production of a surfactant by Candida sphaerica UCP0995. Process Biochemistry. 2008;33:912-917, doi:10.1016/j.procbio.2008.04.013. 